\title{
Allele frequencies of BRAFV600 mutations in primary melanomas and matched metastases and their relevance for BRAF inhibitor therapy in metastatic melanoma
}

\author{
Imke Satzger ${ }^{1}$, Lena Marks ${ }^{1}$, Martin Kerick ${ }^{2}$, Sven Klages ${ }^{2}$, Carola Berking ${ }^{3}$, Rudolf \\ Herbst $^{4}$, Bernward Völker ${ }^{5}$, Vivien Schacht ${ }^{1}$, Bernd Timmermann ${ }^{2}$, Ralf Gutzmer ${ }^{1}$ \\ ${ }^{1}$ Department of Dermatology and Allergy, Skin Cancer Center Hannover, Hannover Medical School, Hannover, Germany \\ ${ }^{2}$ Sequencing Core Facility, Max Planck Institute for Molecular Genetics, Berlin, Germany \\ ${ }^{3}$ Department of Dermatology, Ludwig-Maximilian University of Munich, Munich, Germany \\ ${ }^{4}$ Department of Dermatology and Allergology, HELIOS Skin Cancer Center, Erfurt, Germany \\ ${ }^{5}$ Institute of Pathology, Nordstadt Krankenhaus, Hannover, Germany \\ Correspondence to: \\ Imke Satzger, e-mail: satzger.imke@mh-hannover.de \\ Keywords: metastatic melanoma, primary melanoma, BRAFV600 mutation, BRAF inhibitor \\ Received: March 26, $2015 \quad$ Accepted: October 06, $2015 \quad$ Published: October 16, 2015
}

\section{ABSTRACT}

Background: The detection of BRAFV600 mutations in patients with metastatic melanoma is important because of the availability of BRAF inhibitor therapy. However, the clinical relevance of the frequency of BRAFV600 mutant alleles is unclear.

Patients and Methods: Allele frequencies of BRAFV600 mutations were analyzed by ultra-deep next-generation sequencing in formalin-fixed, paraffin-embedded melanoma tissue (75 primary melanomas and 88 matched metastases). In a second study, pretreatment specimens from 76 patients who received BRAF inhibitors were retrospectively analyzed, and BRAFV600 allele frequencies were correlated with therapeutic results.

Results: Thirty-five patients had concordantly BRAF-positive and 36 (48\%) patients had concordantly BRAF-negative primary melanomas and matched metastases, and four patients had discordant samples with low allele frequencies (3.4-5.2\%). Twenty-six of 35 patients with concordant samples had BRAFV600E mutations, three of whom had additional mutations (V600K in two patients and $V 600 R$ in one) and nine patients had exclusively non-V600E mutations (V600K in eight patients and V6O0E -C.1799_1800TG > AA- in one patient). The frequency of mutated BRAFV600 alleles was similar in the primary melanoma and matched metastasis in 27/35 patients, but differed by $>3$-fold in 8/35 of samples. BRAFV600E allele frequencies in pretreatment tumor specimens were not significantly correlated with treatment outcomes in $\mathbf{7 6}$ patients with metastatic melanoma who were treated with BRAF inhibitors.

Conclusions: BRAFV600 mutation status and allele frequency is consistent in the majority of primary melanomas and matched metastases. A small subgroup of patients has double mutations. BRAFV600 allele frequencies are not correlated with the response to BRAF inhibitors.

\section{INTRODUCTION}

In patients with BRAFV600E-positive melanoma enrolled in large randomized phase III studies, treatment with BRAF kinase inhibitors such as vemurafenib and dabrafenib resulted in response rates of over $50-60 \%$ and progression-free survival (PFS) of 6-7 months. Among nonresponders in these trials, the majority had an initial period of disease stabilization, and only a minority had primary resistance to BRAF inhibitor therapy $[1,2]$. 
Precise selection of patients is crucial for optimal use of BRAF inhibitor therapy. BRAF mutations may be detected in archived formalin-fixed, paraffin-embedded (FFPE) melanoma tissue; however, it is currently unclear whether primary tumors, consecutive metastases, or both should be preferentially analyzed because of the possibility of intertumor heterogeneity [3]. It is also unclear whether the allele frequency of BRAFV600E mutations is correlated with response to BRAF kinase inhibitors. Thus in the first study population, we evaluated BRAFV600 mutations and allele frequencies in FFPE melanoma specimens using ultra-deep next-generation sequencing (NGS) and compared the results in primary melanomas and matched metastases. In a second study population we used NGS to evaluate BRAFV600 mutations in pretreatment melanoma specimens from 76 patients with metastatic melanoma who subsequently received BRAF inhibitors, and examined correlations between BRAFV600 allele frequencies, PFS, overall survival (OS), and objective response.

\section{RESULTS}

BRAFV600 mutational status was determined by ultra-deep NGS in 163 FFPE tissue samples obtained from 75 patients (Table 1 and 2). The primary melanoma and consecutive metastases from one, two, and three

Table 1: Frequencies (\%) of BRAFV600-mutated alleles detected by next-generation sequencing (NGS)

\begin{tabular}{|c|c|c|c|c|c|c|}
\hline Patient number & $\begin{array}{c}\text { Melanoma } \\
\text { sample }\end{array}$ & NGS result & $\operatorname{V600E}(\%)$ & $\operatorname{V600K}(\%)$ & $\operatorname{V600R}(\%)$ & V600E2 (\%) \\
\hline \multirow[t]{2}{*}{1} & $\mathrm{pm}$ & V600E & 18,3 & 0 & 0 & 0 \\
\hline & $\ln$ & V600E & 3,7 & 0 & 0 & 0 \\
\hline \multirow[t]{4}{*}{2} & $\mathrm{pm}$ & V600E & 24,4 & 0 & 0 & 0 \\
\hline & $\ln$ & V600E & 18,7 & 0 & 0 & 0 \\
\hline & $\mathrm{sc}$ & V600E & 24,6 & 0 & 0 & 0 \\
\hline & visceral & V600E & 25,6 & 0 & 0 & 0 \\
\hline \multirow[t]{2}{*}{3} & $\mathrm{pm}$ & V600E & 45,1 & 0 & 0 & 0 \\
\hline & $\mathrm{sc}$ & V600E & 17,7 & 0 & 0 & 0 \\
\hline \multirow[t]{2}{*}{4} & $\mathrm{pm}$ & V600E & 78,5 & 0 & 0 & 0 \\
\hline & $\mathrm{sc}$ & V600E & 86,5 & 0 & 0 & 0 \\
\hline \multirow[t]{2}{*}{5} & $\mathrm{pm}$ & V600E & 38,5 & 0 & 0 & 0 \\
\hline & $\mathrm{Ln}$ & V600E & 45,3 & 0 & 0 & 0 \\
\hline \multirow[t]{2}{*}{6} & $\mathrm{pm}$ & V600E & 40,8 & 0 & 0 & 0 \\
\hline & $\mathrm{sc}$ & V600E & 33,2 & 0 & 0 & 0 \\
\hline \multirow[t]{2}{*}{7} & $\mathrm{pm}$ & V600E & 61,3 & 0 & 0 & 0 \\
\hline & $\mathrm{sc}$ & V600E & 50,9 & 0,1 & 0 & 0 \\
\hline \multirow[t]{2}{*}{8} & $\mathrm{pm}$ & V600E & 9,4 & 0 & 0 & 0 \\
\hline & $\mathrm{sc}$ & V600E & 7,4 & 0 & 0 & 0 \\
\hline \multirow[t]{2}{*}{9} & $\mathrm{pm}$ & V600E & 24,5 & 0 & 0 & 0 \\
\hline & $\mathrm{sc}$ & V600E & 69 & 0 & 0 & 0 \\
\hline \multirow[t]{2}{*}{10} & $\mathrm{pm}$ & V600E & 10,6 & 0 & 0 & 1,8 \\
\hline & $\ln$ & V600E & 8,1 & 0 & 0 & 0 \\
\hline \multirow[t]{3}{*}{11} & $\mathrm{pm}$ & V600E & 31,9 & 0 & 0 & 0 \\
\hline & $\mathrm{sc}$ & V600E & 20,1 & 0 & 0 & 0 \\
\hline & $\ln$ & V600E & 17,3 & 0 & 0 & 0 \\
\hline
\end{tabular}

(Continued) 


\begin{tabular}{|c|c|c|c|c|c|c|}
\hline Patient number & $\begin{array}{c}\text { Melanoma } \\
\text { sample }\end{array}$ & NGS result & $\operatorname{V600E}(\%)$ & $\operatorname{V600K}(\%)$ & $\operatorname{VG00R}(\%)$ & V600E2 (\%) \\
\hline \multirow[t]{2}{*}{12} & $\mathrm{pm}$ & V600E & 23,9 & 0 & 0 & 0 \\
\hline & $\mathrm{sc}$ & V600E & 47,2 & 0 & 0 & 0 \\
\hline \multirow[t]{2}{*}{13} & $\mathrm{pm}$ & V600E & 5 & 0,1 & 0 & 0 \\
\hline & $\ln$ & V600E & 16 & 0 & 0 & 0 \\
\hline \multirow[t]{3}{*}{14} & $\mathrm{pm}$ & V600E & 29 & 0 & 0 & 0 \\
\hline & $\mathrm{sc}$ & V600E & 36,2 & 0 & 0 & 0 \\
\hline & $\ln$ & V600E & 40,7 & 0,1 & 0 & 0 \\
\hline \multirow[t]{2}{*}{15} & $\mathrm{pm}$ & V600E & 21 & 0 & 0 & 0 \\
\hline & $\ln$ & V600E & 10,9 & 0 & 0 & 0 \\
\hline \multirow[t]{2}{*}{16} & $\mathrm{pm}$ & V600E & 51,2 & 0 & 0 & 0 \\
\hline & $\mathrm{sc}$ & V600E & 24,2 & 0 & 0 & 0 \\
\hline \multirow[t]{3}{*}{17} & $\mathrm{pm}$ & V600E & 26,9 & 0 & 0 & 0 \\
\hline & $\mathrm{sc}$ & V600E & 46,6 & 0 & 0 & 0 \\
\hline & $\ln$ & V600E & 58 & 0 & 0 & 0 \\
\hline \multirow[t]{2}{*}{18} & $\mathrm{pm}$ & V600E & 23,4 & 0 & 0 & 0 \\
\hline & $\mathrm{sc}$ & V600E & 54,3 & 0 & 0 & 0 \\
\hline \multirow[t]{3}{*}{19} & $\mathrm{pm}$ & V600E & 44,2 & 0 & 0 & 0 \\
\hline & $\ln$ & V600E & 14,6 & 0 & 0 & 0 \\
\hline & $\mathrm{sc}$ & V600E & 28,3 & 0 & 0 & 0 \\
\hline \multirow[t]{2}{*}{20} & $\mathrm{pm}$ & V600E & 22,4 & 0 & 0 & 0 \\
\hline & $\mathrm{sc}$ & V600E & 7,1 & 0 & 0 & 0 \\
\hline \multirow[t]{2}{*}{21} & $\mathrm{pm}$ & V600E & 7 & 0 & 0 & 0 \\
\hline & $\mathrm{sc}$ & V600E & 49,5 & 0,2 & 0 & 0 \\
\hline \multirow[t]{2}{*}{22} & $\mathrm{pm}$ & V600E & 31,9 & 0 & 0 & 0 \\
\hline & $\ln$ & V600E & 34,7 & 0,2 & 0 & 0 \\
\hline \multirow[t]{2}{*}{23} & $\mathrm{pm}$ & V600E & 28,1 & 0,1 & 0 & 0 \\
\hline & $\mathrm{sc}$ & V600E & 24,4 & 0 & 0 & 0 \\
\hline \multirow[t]{2}{*}{24} & $\mathrm{pm}$ & V600E & 12,7 & 0 & 0 & 0 \\
\hline & $\ln$ & V600E & 92,2 & 0 & 0 & 0 \\
\hline \multirow[t]{2}{*}{25} & $\mathrm{pm}$ & V600E & 23,6 & 0 & 0 & 0 \\
\hline & brain & V600E & 54,2 & 0 & 0 & 0 \\
\hline \multirow[t]{2}{*}{26} & $\mathrm{pm}$ & V600E & 5,6 & 0 & 1,4 & 0 \\
\hline & $\ln$ & $V 600 E+R$ & 20,8 & 0 & 11,4 & 0 \\
\hline \multirow[t]{2}{*}{27} & $\mathrm{pm}$ & V600K & 0,1 & 34 & 0 & 0 \\
\hline & $\mathrm{sc}$ & V600K & 0 & 23,4 & 0 & 0 \\
\hline \multirow[t]{2}{*}{28} & $\mathrm{pm}$ & V600K & 0 & 66,2 & 0,2 & 0 \\
\hline & $\ln$ & V600K & 0 & 9,6 & 0,1 & 0 \\
\hline
\end{tabular}

(Continued) 


\begin{tabular}{|c|c|c|c|c|c|c|}
\hline Patient number & $\begin{array}{c}\text { Melanoma } \\
\text { sample }\end{array}$ & NGS result & V600E $(\%)$ & $\operatorname{V600K}(\%)$ & V600R (\%) & V600E2 (\%) \\
\hline \multirow[t]{2}{*}{29} & $\mathrm{pm}$ & V600K & 0,1 & 44,5 & 0 & 0 \\
\hline & $\ln$ & $V 600 K$ & 0 & 12,7 & 0 & 0 \\
\hline \multirow[t]{3}{*}{30} & $\mathrm{pm}$ & $V 600 K$ & 0 & 46,5 & 0 & 0 \\
\hline & $\ln$ & $V 600 K$ & 0 & 47,8 & 0 & 0 \\
\hline & $\mathrm{sc}$ & $V 600 K$ & 0,2 & 50,7 & 0 & 0 \\
\hline \multirow[t]{2}{*}{31} & $\mathrm{pm}$ & $V 600 K$ & 0,5 & 34,4 & 0 & 0 \\
\hline & $\ln$ & V600K & 0 & 83,7 & 0 & 0 \\
\hline \multirow[t]{2}{*}{32} & $\mathrm{pm}$ & $V 600 K$ & 0 & 32,8 & 0 & 0 \\
\hline & $\mathrm{sc}$ & $V 600 K+E$ & 13,1 & 19,1 & 0 & 0 \\
\hline \multirow[t]{2}{*}{33} & $\mathrm{pm}$ & $V 600 K$ & 0 & 13,2 & 0 & 0 \\
\hline & $\mathrm{sc}$ & $V 600 K+E$ & 5,2 & 14,6 & 0 & 0 \\
\hline \multirow[t]{2}{*}{34} & $\mathrm{pm}$ & V600E2 & 0 & 0 & 0 & 30,2 \\
\hline & $\mathrm{sc}$ & V600E2 & 0,1 & 0 & 0 & 72,9 \\
\hline \multirow[t]{3}{*}{35} & $\mathrm{pm}$ & V600K & 2 & 17,7 & 0 & 0 \\
\hline & $\mathrm{sc}$ & $V 600 K$ & 0 & 45,5 & 0 & 0 \\
\hline & $\mathrm{sc}$ & V600K & 0,1 & 43,4 & 0 & 0 \\
\hline \multirow[t]{2}{*}{36} & $\mathrm{pm}$ & V600E & 5,2 & 0 & 0 & 0 \\
\hline & $\mathrm{sc}$ & wild type & 0,1 & 0 & 0 & 0 \\
\hline \multirow[t]{2}{*}{37} & $\mathrm{pm}$ & V600E & 4,5 & 0 & 0 & 0 \\
\hline & $\mathrm{sc}$ & wild type & 0 & 0 & 0 & 0 \\
\hline \multirow[t]{2}{*}{38} & $\mathrm{pm}$ & wild type & 0 & 0 & 0 & 0 \\
\hline & $\mathrm{sc}$ & V600E & 3,4 & 0 & 0,2 & 0 \\
\hline \multirow[t]{3}{*}{39} & $\mathrm{pm}$ & V600E & 5 & 0 & 0 & 0 \\
\hline & $\mathrm{sc}$ & wild type & 0 & 0 & 0 & 0 \\
\hline & $\mathrm{sc}$ & wild type & 0,8 & 0 & 0 & 0 \\
\hline
\end{tabular}

Three patients $(26,32$, and 33) had double (BRAFV600E and non-BRAFV600E) mutations. NGS allele frequencies $>3 \%$ were considered to be positive $(\mathrm{pm}=$ primary melanoma, $\ln =$ lymph node metastasis, $\mathrm{sc}=$ subcutaneous.

locations were available for 63,11 , and one patient, respectively. In addition to the 75 primary melanoma samples, the analysis included 49 skin metastases, 36 lymph node metastases, two visceral metastases, and one brain metastasis.

Among the 163 tissue samples evaluated, 81 $(50 \%)$ specimens were BRAFV600-negative, 79 (48\%) specimens had a single BRAFV600 mutation. 61 patients had BRAFV600E (c.1799T > A), 16 had BRAFV600K_(c.1798_1799GT >AA) and two patients had BRAFV600E_(c.1799_1800TG >AA), and three $(2 \%)$ specimens had two different BRAFV600 mutations; two had BRAFV600E (c.1799T > A) and BRAFV600K_(c.1798_1799GT > AA) and one had BRAFV600E (c.1799T > A) and BRAFV600R (c.1798_1799GT $>A T_{2}$ Table 1).

\section{Evaluation of BRAF status in primary melanomas and matched metastases by NGS}

Consistent mutation patterns in primary tumors and matched metastatic lesions were observed in 71 of 75 (95\%) patients. A total of 35 patients had concordantly BRAF-positive and 36 (48\%) patients had concordantly BRAF-negative primary melanomas and matched metastases The four $(5 \%)$ remaining patients each had one BRAFV600-positive and one BRAFV600-negative sample. In three of these four patients, the primary melanoma was 
Table 2: Clinical characterization of 75 patients; 75 primary melanomas of these patients and 88 matched metastases were analyzed by ultra-deep next generation sequencing to compare BRAF V600 status and BRAF V600 allele frequencies of primary melanomas and matched metastases

\begin{tabular}{|l|l|c|}
\hline Clinical parameters & N & $75(100 \%)$ \\
\hline Total patients & Male & $46(61 \%)$ \\
\hline Gender & Female & $29(39 \%)$ \\
\hline & Mean & 61 \\
\hline Age (years) & Median & 65 \\
\hline & Minimum-maximum & $29-91$ \\
\hline & & \\
\hline BRAF status & Primary melanoma & 75 \\
\hline Tissue tested & Lymph node metastases & 36 \\
\hline & Cutaneous metastases & 49 \\
\hline & Visceral metastases & 2 \\
\hline & Brain metastases & 1 \\
\hline
\end{tabular}

BRAFV600-positive, and in the one remaining patient the metastatic tissue alone was BRAFV600-positive (Table 1). Of note, the BRAFV600 allele frequencies were low (3.4-5.2\%) in the positive samples from these four individuals (Table 1).

\section{BRAFV600E (c.1799T > A) mutations and rare mutations by NGS}

Among the 35 patients with concordantly BRAFpositive samples, 26 patients had a BRAFV600E $(c .1799 T>A)$ mutation in both the primary melanoma and consecutive metastases, eight patients had BRAF V600K (c.1798_1799GT > AA) mutations (eight primary melanomas, four lymph node metastases, and four skin metastases), and one patient had a BRAFV600E (c.1799_1800TG $>A A$ ) mutation (in the primary melanoma and matched skin metastasis) (Table 1). Three metastatic specimens from these 35 patients (two skin metastases and one lymph node metastasis) showed BRAFV600K (c.1798_1799GT > AA, $n=2$ ) or BRAFV600R (c.1798_1799GT >AT; $\mathrm{n}=1$ ) mutations with an allele frequency $>3 \%$, in addition to BRAFV600E (c.1799T $>A)$.

\section{Frequencies of mutated BRAFV600 alleles in primary melanomas and matched metastases by NGS}

The median percentage of mutated alleles was $28 \%$ in primary melanomas and $26 \%$ in consecutive metastases (Figure 1). The mutant allele frequency was higher in the primary melanoma than in the metastases in 16 patients, and higher in the metastases than the primary melanoma in 19 patients (Table 1, Figure 1). In 27 of 35 (77\%) patients with BRAFV600 mutations, the percentage of mutated alleles in the primary melanoma and metastases differed by $<3$-fold. In the eight patients in whom the percentage of mutated alleles in the primary melanoma and metastases differed by $>3$-fold, the frequencies of mutated alleles was higher in the primary melanoma in four patients and higher in the metastases in four patients. The differences in allele frequencies between primary and metastatic tissue in six of these eight patients could be attributed to differences in tumor cell content in the various tissues.

\section{Allele frequencies of patients treated with BRAF inhibitors and their impact on therapy outcome}

Pretreatment samples from 76 patients with BRAFV600E-positive metastatic melanoma who were treated with the BRAF inhibitors vemurafenib $(n=67)$ or dabrafenib $(n=9)$ were retrospectively analyzed by NGS. The baseline characteristics and response to therapy after a mean followup of 11.4 months are summarized in Table 3. The available samples included nine primary melanomas, 29 lymph node metastases, 28 cutaneous or subcutaneous metastases, eight visceral metastases, and two brain metastases. As shown in Table 3, BRAFV600E (c.1799T > A) allele frequencies in pretreatment melanoma tissue were $\leq 5 \%$ in two patients, $>5$ $10 \%$ in four patients, $>10-15 \%$ in three patients, $>15-20 \%$ in 11 patients, $>20-25 \%$ in 6 patients, $>25-50 \%$ in 33 patients, and $>50 \%$ in 17 patients.

Allele frequencies were not significantly correlated with either PFS or OS in Kaplan-Meier 


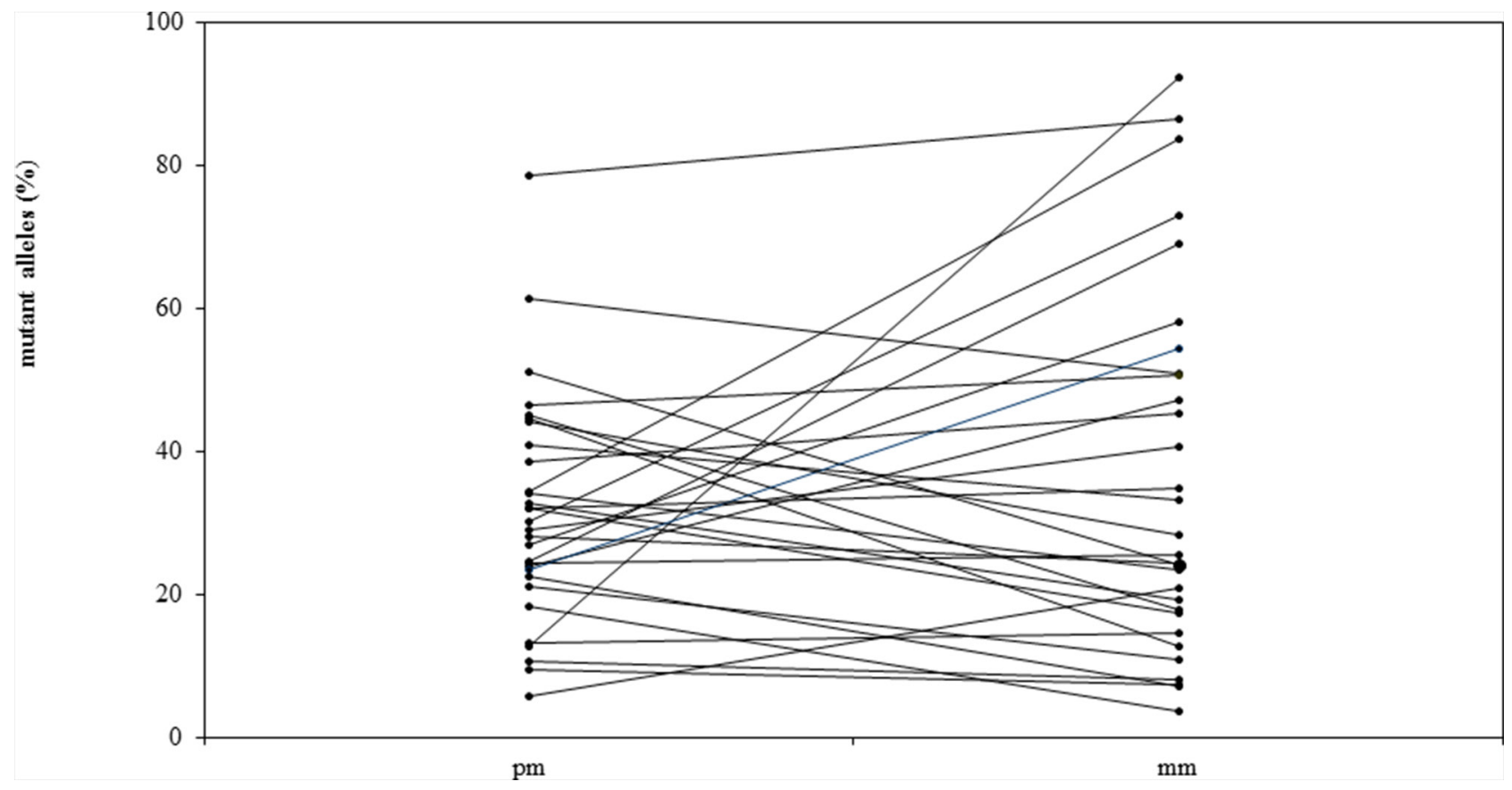

Figure 1: Allele frequencies (\%) of BRAFV600 mutations in primary melanomas (pm) and matched metastases (mm) in 35 patients with metastatic melanoma.

analyses in which different cut-offs were used $(\leq 15 \%$, $\leq 18 \%, \leq 20 \%$, and $\leq 25 \%$ ). Comparisons of PFS and OS in patients with allele frequencies $\leq 18$ and $>18 \%$ are shown in Figure 2 ( $p=0.374$ for PFS and $p=0.898$ for OS). Odds Ratio was calculated to determine the magnitude of differences that can be detected with this relatively small cohort (Table 4).

The response to therapy (complete response [CR] and partial response $[\mathrm{PR}]$ versus stable disease $[\mathrm{SD}]$, mixed response $[\mathrm{MR}]$, and progressive disease [PD]) was not significantly correlated with allele frequencies in univariate analyses in which different cutoffs were used $(15 \%, 18 \%$, $20 \%$, and $25 \%$ ). A total of $9,15,20$, and 26 patients had BRAF V600E allele frequencies $\leq 15 \%, \leq 18 \%, \leq 20 \%$, and $\leq 25 \%$, respectively. Response rates (CR and $\mathrm{PR}$ ) in these categories were as follows: BRAFV600E allele frequency $\leq 15 \%$ versus $>15 \%(44 \%$ and $61 \%, p=0.473) ; \leq 18 \%$ versus $>18 \%(40 \%$ and $64 \%, p=0.142) ; \leq 20 \%$ versus $>20 \%(45 \%$ and $64 \%, p=0.185)$; and $\leq 25 \%$ versus $>25 \%$ $(50 \%$ and $64 \%, p=0.326)$.

\section{DISCUSSION}

Since the discovery of BRAF mutations in melanoma in 2002 [4], advances in molecular characterization of the disease have led to the development of specific BRAF inhibitors that are now available for the treatment of patients with metastatic melanoma. In the first part of this study, we used ultra-deep NGS to assess the BRAFV600 mutation status of primary melanomas and matched metastases and their influence on the outcome of BRAF inhibitor therapy.

For the first time, we showed that type and frequency of BRAFV600 mutations are consistent in the primary melanoma and matched metastases in the majority of patients. In most patients with discordant BRAFV600 status, in which one tissue sample was positive and one sample negative, this could be explained by low allele frequencies where the allele frequency was close to the threshold of detection in the positive sample but below the threshold of detection in the negative sample. Differences in allele frequencies could also be explained by differences in tumor cell content in the specimens. These results suggest that, in the majority of melanoma cases, the BRAF mutation status of the primary tumor is retained in metastases, and that primary and/or metastatic tissue can be used for routine mutational analysis provided that sufficient tumor cell content is available.

Five recently published studies have analyzed the BRAF mutation status of melanoma samples with different molecular methods (Table 5). Consistent with our observations, these analyses also found the mutational status of primary tumor and metastatic tissue to be concordant in the majority of cases. Intrapatient homogeneity of BRAF mutations has also been reported in all patients $(n=64)$ included in a recent study that used immunohistochemical methods for detection of BRAF mutants [5].

Our findings suggest that melanomas can be heterogeneous with regard to BRAFV600 mutations. The allele frequency was below $50 \%$, which would be the 
Table 3: Characterization of 76 patients with BRAFV600E mutations who were treated with BRAF inhibitors for metastatic melanoma

\begin{tabular}{|c|c|c|}
\hline \multicolumn{3}{|l|}{ Clinical parameters } \\
\hline Total patients & $N$ & $76(100 \%)$ \\
\hline \multirow[t]{2}{*}{ Gender } & Male & $41(54 \%)$ \\
\hline & Female & $35(46 \%)$ \\
\hline \multirow[t]{3}{*}{ Age (years) } & Mean & 56 \\
\hline & Median & 60 \\
\hline & Minimum-maximum & $13-84$ \\
\hline \multicolumn{3}{|l|}{ BRAF status } \\
\hline \multirow[t]{5}{*}{ Tissue tested } & Primary melanoma & $9(12 \%)$ \\
\hline & Lymph node metastases & $29(38 \%)$ \\
\hline & Cutaneous metastases & $28(37 \%)$ \\
\hline & Visceral metastases & $8(10 \%)$ \\
\hline & Brain metastases & $2(3 \%)$ \\
\hline Allele frequencies & Mean & 31.9 \\
\hline \multirow[t]{9}{*}{ BRAFV600E mutation (\%) } & Median & 34.1 \\
\hline & Minimum-Maximum & $3.7-81.2$ \\
\hline & $\leq 5$ & $2(3 \%)$ \\
\hline & $>5-10$ & $4(5 \%)$ \\
\hline & $>10-15$ & $3(4 \%)$ \\
\hline & $>15-20$ & $11(15 \%)$ \\
\hline & $>20-25$ & $6(8 \%)$ \\
\hline & $>25-50$ & $33(43 \%)$ \\
\hline & $>50$ & $17(22 \%)$ \\
\hline \multicolumn{3}{|l|}{ Treatment } \\
\hline \multirow[t]{2}{*}{ BRAF inhibitor } & Vemurafenib & $67(88 \%)$ \\
\hline & Dabrafenib & $9(12 \%)$ \\
\hline \multirow[t]{5}{*}{ Prior therapies for metastatic melanoma } & No & $44(57 \%)$ \\
\hline & 1 prior therapy & $12(16 \%)$ \\
\hline & 2 prior therapies & $14(19 \%)$ \\
\hline & 3 prior therapies & $3(4 \%)$ \\
\hline & $\geq 4$ prior therapies & $3(4 \%)$ \\
\hline \multicolumn{3}{|l|}{ Response to treatment } \\
\hline \multirow[t]{5}{*}{ Best response } & $\mathrm{CR}$ & $5(7 \%)$ \\
\hline & PR & $40(53 \%)$ \\
\hline & SD & $17(22 \%)$ \\
\hline & MR & $10(13 \%)$ \\
\hline & PD & $4(5 \%)$ \\
\hline
\end{tabular}

(Continued) 


\begin{tabular}{|l|l|c|}
\hline Time to progression (months) & Mean & 5.5 \\
\hline & Median & 7.1 \\
\hline & Minimum-maximum & $0.5-26.1$ \\
\hline Progression & No & $15(20 \%)$ \\
\hline & Yes & $61(80 \%)$ \\
\hline Follow-up & \multicolumn{2}{|c|}{} \\
\hline Death & No & $31(41 \%)$ \\
\hline & Yes, due to melanoma & $41(54 \%)$ \\
\hline & Yes, other cause & $4(5 \%)$ \\
\hline Follow-up (months) & Mean & 11.4 \\
\hline & Median & 9.7 \\
\hline & Minimum-maximum & $0.8-27.8$ \\
\hline
\end{tabular}

$(\mathrm{sc}=$ subcutaneous, $\mathrm{CR}=$ complete response, $\mathrm{MR}=$ mixed response, $\mathrm{PD}=$ progressive disease, $\mathrm{PR}=$ partial response, $\mathrm{SD}=$ stable disease).
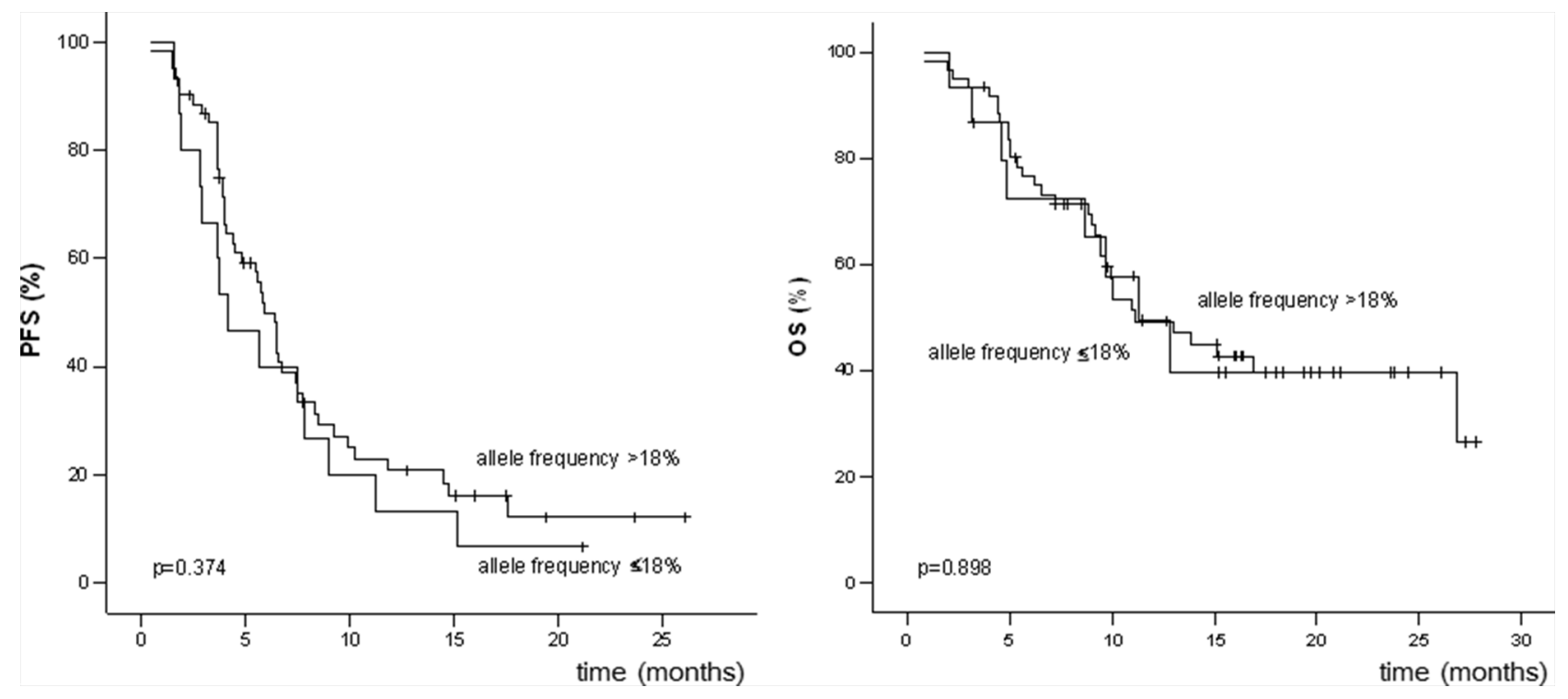

Figure 2: Progression-free survival (PFS) and overall survival (OS) of patients with BRAFV600 allele frequencies $\leq 18 \%$ and $>18 \%$.

expected result if cells show a consistent heterozygous mutation. For example, patient number 2 showed allele frequencies that ranged from $18.7 \%$ to $25.6 \%$ in the primary tumor and three metastatic samples (Table 1). The results of a study that used a BRAFV600E-specific antibody and showed heterogeneous staining in 13 of 58 (22\%) melanoma samples provides further evidence of heterogeneity of BRAFV600 mutations [6]. Moreover, a further study that used single cell suspensions to assess BRAF mutations found 9 of 10 primary melanomas and 0 of 3 metastases to be heterogeneous [7].

Despite these data that suggest BRAFV600 mutations are heterogeneous, the clinical response to
BRAF inhibitors is homogenous $[1,2]$ and suggests that the BRAFV600 mutation is relevant in the majority of melanoma cells.

Approximately $10 \%$ of patients with BRAF V600 mutations had double mutations, comprised of the BRAFV600E and an additional rare mutation. This phenomenon has not been reported previously and demonstrates the ability of NGS to detect different mutations that are difficult or impossible to detect with techniques used in other studies, such as Sanger sequencing, pyrosequencing or melting curve analyses $[3,8-11]$. This phenomenon may also be the result of intratumor heterogeneity. 
Table 4: Required 95\% confidence interval limits for statistical significance in response analysis

\begin{tabular}{|l|c|c|c|c|}
\cline { 2 - 5 } \multicolumn{1}{c}{ Parameters } & $\mathbf{1 5 \%}$ & $\mathbf{1 8 \%}$ & $\mathbf{2 0 \%}$ & $\mathbf{2 5 \%}$ \\
\hline Proportion $\pi_{1}$ & $15 \%$ & $25 \%$ & $28 \%$ & $31 \%$ \\
\hline${\text { Proportion } \pi_{2}}^{n}$ & $61 \%$ & $64 \%$ & $64 \%$ & $64 \%$ \\
\hline Odds ratio $^{1}$ & 8.86 & 5.33 & 4.57 & 3.96 \\
\hline${\text { Distance }{ }^{2}}^{2}$ & $\mathbf{1 . 8 9}$ & $\mathbf{1 . 2 8}$ & $\mathbf{1 . 1 2}$ & $\mathbf{1 . 0 1}$ \\
\hline Lower limit $^{3}$ & $\mathbf{1 . 3 3}$ & $\mathbf{1 . 4 8}$ & $\mathbf{1 . 4 9}$ & $\mathbf{1 . 4 4}$ \\
\hline Upper limit $^{3}$ & $\mathbf{5 8 . 9 3}$ & $\mathbf{1 9 . 1 9}$ & $\mathbf{1 3 . 9 9}$ & $\mathbf{1 0 . 8 9}$ \\
\hline
\end{tabular}

Note: $\pi 1$ : proportion of responders with BRAFV600E allele frequency $\leq$ cut-off; $\pi 2$ : proportion of responders with BRAFV600E allele frequency $>$ cut-off.

${ }^{1}$ This is the minimum required effect size estimated for power equal to $80 \%$ (proportion of responders with BRAFV600E allele frequency $>$ cut-off against proportion of responders with BRAFV600E allele frequency $\leq$ cut-off).

${ }^{2}$ Distance refers to the difference between the logarithm of odds ratio and its lower limit.

${ }^{3}$ Results are presented in the exponential (original) scale.

\section{Table 5: Evaluation of BRAFV600 mutations in primary melanomas and matched metastases by different molecular detection methods}

\begin{tabular}{|c|c|c|c|}
\hline Detection method & Tumor cell content $(\%)$ & Concordance rate, $\mathrm{n} / \mathrm{N}(\%)$ & Ref. \\
\hline NGS & $>30$ & $71 / 75(95)$ & This study \\
\hline $\begin{array}{l}\mathrm{HRM}+\text { direct sequencing } \\
\text { (Sanger) }\end{array}$ & $>10$ & $84 / 88(95)$ & (12) \\
\hline $\begin{array}{l}\text { MS-PCR + direct sequencing } \\
\text { (Sanger) }\end{array}$ & $<33$ to $>67$ & $10 / 18(56)$ & (3) \\
\hline Pyrosequencing & $>75$ & $43 / 53(81)$ & (14) \\
\hline Direct sequencing (Sanger) & NS & $21 / 24(87)$ & (13) \\
\hline Direct sequencing (Sanger) & $\geq 80$ & $87 / 102(85)$ & (16) \\
\hline
\end{tabular}

$(\mathrm{HRM}=$ high-resolution melting curve analysis, $\mathrm{MS}-\mathrm{PCR}=$ mutagenically separated polymerase chain reaction, $\mathrm{NGS}=$ next-generation sequencing, NS = not stated)

Allele frequencies had no impact on PFS, OS, and objective response rates in the 76 patients with BRAFV600E-positive metastatic melanoma treated with BRAF inhibitors. Although the usual precautions regarding retrospective analyses apply, the median PFS of 7.1 months and the response rate of $60 \%$ are very close to the results of the phase III studies [1,2], supporting the validity of our findings. These results are also in line with the recently published observation of Wilmott et al. that the intensity and distribution of BRAFV600E immunohistochemical staining is not correlated with clinical outcomes [6].

The observation that patients with allele frequencies $<18 \%$ and $\geq 18 \%$ have similar PFS and OS is clinically relevant, because an allele frequency of $18 \%$ is around the detection limit of Sanger sequencing [12], which is commonly used in routine clinical practice. Thus, in the case of a negative Sanger sequencing result, our data suggest that retesting with a more sensitive assay may be worthwhile to detect alleles that may be present at lower frequencies.

In conclusion, we show here that BRAFV600 mutation status and allele frequency are consistent in the majority of primary melanomas and matched metastases, that a subgroup of patients has double mutations, and that the allele frequency of melanoma tissue is not correlated with treatment response in our patient cohort. 


\section{MATERIALS AND METHODS}

\section{Patients}

In the first study population we retrospectively analyzed melanoma samples from patients with metastatic melanoma who were treated at the Skin Cancer Center Hannover, Germany, between 1995 and 2011. Patients were selected for this study on the basis of availability of primary melanoma tissue and tissue from at least one matched metastasis.

In a second different study population, BRAFV600 allele frequencies were determined by NGS in pretreatment melanoma tissue specimens from metastatic melanoma patients who were treated with BRAF inhibitors at three German skin cancer centers (Munich, Erfurt and Hannover). Patients had been treated with standard dosages of vemurafenib (960 mg b.i.d.) in a phase III clinical trial [13] or expanded access program, or dabrafenib (150 mg b.i.d.) in phase III clinical trials [14]. Only patients with BRAFV600E mutations and with pretreatment FFPE tumor specimens available for NGS analysis were included. Patients with non-V600E mutations and patients with double mutations were excluded.

Response to therapy in the clinical trials was assessed with computed tomography every $8-12$ weeks, as required by the protocols $[13,14]$. Tumor responses were determined according to Response Evaluation Criteria In Solid Tumors (RECIST) version 1.1 [15].

\section{Histopathology, macrodissection, DNA extraction}

One slide at the beginning of each serial section was stained with hematoxylin-eosin and histopathologically examined to determine the tumor cell content. Only samples with a tumor cell content of at least $30 \%$ were included in this study. The area of interest was circled on the stained slide and macrodissection was performed on the corresponding unstained slides using a scalpel. DNA was extracted using the cobas ${ }^{\circledR}$ DNA Sample Preparation Kit (Roche, Grenzach-Wyhlen, Germany).

\section{Ultra-deep NGS}

Isolated DNA (350-976 ng) from all FFPE specimens was subjected to analyses by NGS using a Roche GS Junior System to detect BRAFV600 mutations on exon 15. The NGS procedure was done according to the manufacturer's specifications [16]. Amplicon processing, library preparation and emulsion PCR were done according to the manufacturer's directions for the GS Junior Titanium Series (Roche). Around 500,000 enriched beads were loaded on a 454 Junior Sequencer (Roche, Basel, Switzerland). Demultiplexing and variant calling was done with the Amplicon Variant Analyzer v2.7 software from Roche. The average sequencing coverage of BRAF was $>5000$. The presence of a BRAFV 600 mutation was defined as the presence of a non-reference base in a minimum of $3 \%$ of reads.

\section{Approval by ethics committee}

The collection of clinical and follow-up data, performance of mutational analyses, and correlation with clinical data was approved by the Ethics Committee of Hannover Medical School (vote 1849-2013).

\section{Statistical analyses}

Associations between BRAFV600E allele frequencies and clinical outcomes were tested by logrank test (Kaplan-Meier analyses) using different allele frequencies as cutoffs $(15 \%, 18 \%, 20 \%$, and $25 \%)$. Qualitative comparisons of objective response to therapy with BRAF inhibitors were performed using RECIST 1.1 criteria. In these comparisons, responders were defined as having either a CR or PR and nonresponders were defined as having SD, an MR, or PD. Analyses of responses to therapy (response versus nonresponse) and allele frequencies were performed by using a two-tailed Fisher's exact test. Statistical significance was defined as an alpha level $<0.05$. SPSS 19.0 (SPSS Inc; Chicago, IL, USA) was used for Kaplan-Meier tests, Fisher's exact test and calculation of Odds ratio (OR).

\section{ACKNOWLEDGMENTS AND FUNDING}

The assistance of Nadine Went, Andre Meier and Loukia Spineli is gratefully acknowledged. We thank Health Interactions for writing support.

Supported by Grant from Roche Pharma AG, Grenzach-Wyhlen, Germany. The funding body had no role in the design or conduct of the study.

Dr. Satzger has received payments for serving on scientific advisory boards for Roche and BristolMyers Squibb, and grant support from Roche, Pfizer, and Novartis; Dr. Gutzmer has received consulting fees and honoraria for lectures from Bristol-Myers Squibb, GlaxoSmithKline, Merck-Serono, MSD, Novartis, Roche, Janssen, Amgen, Almirall-Hermal, and Pfizer. Dr. Berking has received consulting fees, honoraria for lectures and/ or travel support by AstraZeneca, Bristol-Myers Squibb, GlaxoSmithKline, MSD, Novartis, and Roche. Dr. Herbst has received consulting fees, honoraria for lectures and travel support for Bristol-Myers Squibb, Merck-Serono, GlaxoSmithKline, and Roche. 


\section{CONFLICTS OF INTEREST}

No other authors have any relevant conflict of interest.

\section{REFERENCES}

1. Hauschild A, Grob JJ, Demidov LV, Jouary $T$, Gutzmer R, Millward M, Rutkowski P, Blank CU, Miller WH, Kaempgen E, Martin-Algarra S, Karaszewska B, Mauch C, et al. An update on BREAK3, a phase III, randomized trial: Dabrafenib (DAB) versus dacarbazine (DTIC) in patients with BRAF V600E-positive mutation metastatic melanoma (MM). J Clin Oncol. 2013; 31 Abstract 9013 (poster presentation).

2. Chapman PB, Hauschild A, Robert C, Larkin JMG, Haanen JBAG, Ribas A, Hogg D, Hamid O, Ascierto PA, Testori A, Lorigan P, Dummer R, Sosman JA, et al. Updated overall survival (OS) results for BRIM-3, a phase III randomized, open-label, multicenter trial comparing BRAF inhibitor vemurafenib (vem) with dacarbazine (DTIC) in previously untreated patients with BRAF ${ }^{V 600 E}$-mutated melanoma. J Clin Oncol. 2012; 30 Abstract 8502 (oral presentation).

3. Yancovitz M, Litterman A, Yoon J, Ng E, Shapiro RL, Berman RS, Pavlick AC, Darvishian F, Christos P, Mazumdar M, Osman I, Polsky D. Intra- and inter-tumor heterogeneity of BRAF(V600E)) mutations in primary and metastatic melanoma. PLoS One. 2012; 7:e29336.

4. Davies H, Bignell GR, Cox C, Stephens P, Edkins S, Clegg S, Teague J, Woffendin H, Garnett MJ, Bottomley W, Davis N, Dicks E, Ewing R, et al. Mutations of the BRAF gene in human cancer. Nature. 2002; 417:949-954.

5. Menzies AM, Lum T, Wilmott JS, Hyman J, Kefford RF, Thompson JF, O'Toole S, Long GV, Scolyer RA. Intrapatient homogeneity of BRAFV600E expression in melanoma. Am J Surg Pathol. 2014; 38:377-382.

6. Wilmott JS, Menzies AM, Haydu LE, Capper D, Preusser M, Zhang YE, Thompson JF, Kefford RF, von Deimling A, Scolyer RA, Long GV. BRAF(V600E) protein expression and outcome from BRAF inhibitor treatment in BRAF(V600E) metastatic melanoma. Br J Cancer. 2013; 108:924-931.

7. Lin J, Goto Y, Murata H, Sakaizawa K, Uchiyama A, Saida T, Takata M. Polyclonality of BRAF mutations in primary melanoma and the selection of mutant alleles during progression. Br J Cancer. 2011; 104:464-468.
8. Boursault L, Haddad V, Vergier B, Cappellen D, Verdon S, Bellocq JP, Jouary T, Merlio JP. Tumor homogeneity between primary and metastatic sites for BRAF status in metastatic melanoma determined by immunohistochemical and molecular testing. PLoS One. 2013; 8:e70826.

9. Houben R, Becker JC, Kappel A, Terheyden P, Brocker EB, Goetz R, Rapp UR. Constitutive activation of the ras-raf signaling pathway in metastatic melanoma is associated with poor prognosis. J Carcinog. 2004; 3:6.

10. Heinzerling L, Kuhnapfel S, Meckbach D, Baiter M, Kaempgen E, Keikavoussi P, Schuler G, Agaimy A, Bauer J, Hartmann A, Kiesewetter F, Schneider-Stock R. Rare BRAF mutations in melanoma patients: Implications for molecular testing in clinical practice. Br J Cancer. 2013; 108:2164-2171.

11. Colombino M, Capone M, Lissia A, Cossu A, Rubino C, De Giorgi V, Massi D, Fonsatti E, Staibano S, Nappi O, Pagani E, Casula M, Manca A, et al. BRAF/NRAS mutation frequencies among primary tumors and metastases in patients with melanoma. J Clin Oncol. 2012; 30:2522-2529.

12. Querings S, Altmuller J, Ansen S, Zander T, Seidel D, Gabler F, Peifer M, Markert E, Stemshorn K, Timmermann B, Saal B, Klose S, Ernestus K, et al. Benchmarking of mutation diagnostics in clinical lung cancer specimens. PLoS One. 2011; 6:e19601.

13. Chapman PB, Hauschild A, Robert C, Haanen JB, Ascierto P, Larkin J, Dummer R, Garbe C, Testori A, Maio M, Hogg D, Lorigan P, Lebbe C, et al. Improved survival with vemurafenib in melanoma with BRAF V600E mutation. N Engl J Med. 2011; 364:2507-2516.

14. Hauschild A, Grob JJ, Demidov LV, Jouary T, Gutzmer R, Millward M, Rutkowski P, Blank CU, Miller WH Jr, Kaempgen E, Martin-Algarra S, Karaszewska B, Mauch C, et al. Dabrafenib in BRAF-mutated metastatic melanoma: A multicentre, open-label, phase 3 randomised controlled trial. Lancet. 2012; 380:358-365.

15. Eisenhauer EA, Therasse P, Bogaerts J, Schwartz LH, Sargent D, Ford R, Dancey J, Arbuck S, Gwyther S, Mooney M, Rubinstein L, Shankar L, Dodd L, et al. New response evaluation criteria in solid tumours: Revised RECIST guideline (version 1.1). Eur J Cancer. 2009; 45:228-247.

16. Rechsteiner M, von Teichman A, Rüschoff JH, Fankhauser N, Pestalozzi B, Schraml P, Weber A, Wild P, Zimmermann D, Moch H. KRAS, BRAF, and TP53 deep sequencing for colorectal carcinoma patient diagnostics. J Mol Diagn. 2013; 15:299-311. 